\title{
MORPHOLOGY OF THE GENUS GYMNOCEPHALUS (PISCES) FROM THE LOWER DANUBE RIVER
}

\author{
Cecilia ȘERBAN* and Gabriela GRIGORAŞ ** \\ * "Dunărea de Jos" University, Domnească Street 111, RO-800201 Galați, Romania, \\ cecilia.serban@ugal.ro \\ ** "Răsvan Angheluță" Museum Complex of Natural Sciences, Regiment 11 Siret Street, 6A, \\ RO-800340 Galaţi, Romania, griggabi@yahoo.com
}

DOI: 10.2478/trser-2019-0012

KEYWORDS: genus Gymnocephalus, phenotypic variability, morphometric, geometrics, lower Danube.

\section{ABSTRACT}

The current study completed the information regarding the phenotypic variability in the Danube ruffe (Gymnocephalus baloni). We also assessed the phylogenetic relationship of G. baloni with the other two species of the genus Gymnocephalus from the Lower Danube River.

Ten morphological characters were the most useful together for discriminating between G. baloni and G. cernua from the Lower Danube River. In addition, we found a more streamlined body shape in G. baloni compared with the described holotype, which could be in connection with fish phenotypic response to ecological characteristics of the Lower Danube River. Danube.

RESUMÉ: Morphologie du genre Gymnocephalus (Pisces) du cours inférieur du

L'étude actuelle a complété l'information concernant la variabilité phénotypique de la ruffe du Danube (Gymnocephalus baloni). On a également évalué la relation phylogénétique de G. baloni avec les deux autres espèces du genre Gymnocephalus du cours inférieur du Danube.

Dix caractères morphologiques ont été les plus utiles pour différencier $G$. baloni et $G$. cernua du cours inférieur du Danube. De même, on a trouvé une forme de corps plus hydrodynamique chez G. baloni par rapport à l'holotype décrit, ce qui pourrait être en rapport avec la réponse phénotypique des poissons aux caractéristiques écologiques du cours inférieur du Danube. Dunării.

REZUMAT: Morfologia genului Gymnocephalus (Pisces) din cursul inferior al

Studiul a completat informațiile privind variabilitatea fenotipică a ghiborțului de Dunăre (Gymnocephalus baloni). S-a evaluat, de asemenea, relația filogenetică dintre $G$. baloni și celelalte două specii din genul Gymnocephalus din cursul inferior al Dunării.

Zece caractere morfologice au fost semnificative pentru discriminarea speciilor $G$. baloni și G. cernua din cursul inferior al Dunării. De asemenea, s-a evidențiat forma hidrodinamică mai accentuată a speciei G. baloni comparativ cu holotipul, aspect care ar putea fi în legătură cu răspunsul fenotipic al peștilor la caracteristicile ecologice ale fluviului Dunărea în cursul inferior. 


\section{INTRODUCTION}

The highly diversified and complex Lower Danube River area geoecosystems, generated in time and space by a unique mixture of biotopes and biocoenosis, has formed a highly dynamic and extremely rich fish fauna (e.g. there are 137 fish species only in the Danube Delta) (Antipa, 1909; Bănărescu, 1964; Bănărescu and Bănăduc, 2007; Bănăduc et al., 2016).

In the last century, compared to Antipa (1905, 1909, 1915, 1933, 1934), the Lower Danube-Danube Delta-Black Sea's area habitats heterogeneity and native economic and conservation important fish species diversity and stock abundance have a significantly decreasing trend, and currently, there is no scientific indication that this trend will stop in the near future (Bănăduc et al., 2016). In this present context, it is evident that the regional fish species of conservative and/or economic interest should be studied from a high number of points of view.

Three species of the genus Gymnocephalus are widespread in the Lower Danube River basin: the widespread ruffe, Gymnocephalus cernua (Linnaeus, 1758), the Danube ruffe, Gymnocephalus baloni (Holčík and Hensel, 1974) and the yellow pope, Gymnocephalus schraetser (Linnaeus 1758), the last two of these Eurasian fish species being indexed in the Romanian Vertebrate Red List as vulnerable (Bănărescu, 2005) and are protected by Habitats Directive - 92/43/EEC (Bănărescu and Bănăduc, 2007). There is another species of ruffe reported in scientific studies from the Black Sea basin, the Don ruffe, Gymnocephalus acerina (Gmelin, 1789) but the information about its presence in the Prut River, a tributary of the Danube River has not been confirmed by some recent ichthyological studies (Davideanu et al., 2008).

The species from the Eurasian genus Gymnocephalus date to about 13.4 Mya (Stepien and Haponski, 2015). They show different patterns in body shape, colours and meristic characters as lateral line scale and fin rays counts (Bănărescu, 1964; Holčík and Hensel, 1974; Kottelat and Freyhof, 2007; Stepien and Haponski, 2015).

Gymnocephalus baloni has long been treated as G. cernua and just recently described as a specific rank by Holčík and Hensel (1974), the species being poorly known (Bănărescu, 2005; Ratschan, 2012). After its first description, several ichthyological studies reported the first presence of populations in different parts of the Danube River drainage such as the Drava River, Slovenia and Croatia (Povž et al., 1997), and the Mur River, Austria (Ratschan, 2012). A recent study of Tsyba and Kogodiy (2017) notices the presence of the species in another river from Black Sea basin (middle Dniepr/Nistru drainage, Ukraina).

There are several studies from recent years that report $G$. baloni species from different rivers in the Romanian Danube River drainage area: the Danube River - downstream to the town of Brăila, including the Danube Delta arms, the Danube River between Turnu-Severin and Moldova Nouă, as well as the rivers Criş, Someş, Mureş, Ialomiţa, Argeş, Olt, Vedea, Timiş, Tisoviţa, and Berzasca (Oțel et al., 1993; Kováč, 1994; Bănărescu and Bănăduc, 2007; Oțel, 2007; Bănăduc, 2009; Telcean and Cupșa, 2009, 2012; Oțel and Năstase, 2010; Györe et al., 2013; Bănăduc et al., 2014, 2016; Nuță et al., 2016; Năstase and Oțel, 2016; Bulat, 2017; Năstase et al., 2017). 
However, no publications are known to us to describe the phenotypic variability of populations from the Romanian Danube River basin.

The main aim of our research was to analyse the morphometric features of $G$. baloni from the targeted area and compare them with data published by other authors in recent years, regarding the fish populations from the Danube River basin (Bănărescu, 1964; Holčík and Hensel, 1974; Specziár and Vida, 1995; Kottelat and Freyhof, 2007; Geiger and Schliewen, 2010; Stepien and Haponski, 2015; Tsyba and Kokodiy, 2017).

The intra- and inter-population morphological variations in fish are put in evidence by several authors (Karakousis et al., 1991; Wimberger, 1992; Turan, 1999; Shao et al., 2007). Various references sustain the habitat-associated morphological divergence as a common pattern in fish (Foster et al., 2015).

The morphological characters for reliable discrimination between two of these sympatric species are also analysed in our study. An analysis of phenotypic variation in morphometric or meristic characters is the method most commonly used for fish population delineation (Karakousis et al., 1991; Shao et al., 2007; Samaradivakara et al., 2012). This approach is still commonly used in fish biology, taxonomic studies and assessment of the relationship between various taxa (Turan, 1999), despite the development of molecular techniques (Shao et al., 2007).

Another goal of our research was to analyse the phylogenetic relationship between the sister taxa G. cernua and G. baloni from the targeted area. Although there is a strong similarity between G. baloni and G. cernua and a clear discrimination of these two species from $G$. schraetser (with more hydrodynamic shape of the body and also specific colour features), there are still different opinions expressed in the studies of last decades, regarding their phylogenetic relationship (Specziár and Vida, 1995; Geiger and Schliewen, 2010).

Thus, the first taxonomical description of G. baloni by Holčík and Hensel (1974) allowed the authors to identify a closer relationship between G. baloni and G. cernua, placing them in the same subgenus, Acerina, while the remaining two elongated species (G. schraetser and G. acerina) are placed in the subgenus Gymnocephalus. Later, Geiger and Schliewen (2010), using genetic techniques, have questioned the phylogenetic position of G. schraetser within the genus Gymnocephalus, while the new results of Stepien and Haponski (2015), based on molecular data, have suggested a sister - taxa relation between G. baloni and G. schraetser.

\section{MATERIAL AND METHODS}

The morphometric data are from a total of 49 fish specimens in the Lower Danube River area, of them: 12 specimens of the Danube ruffe, G. baloni, and 16 specimens of the striped ruffe, $G$. schraetser were sampled near the confluences of the Danube River with the rivers Prut and Siret at Galați City (sea mile 80-76 from Black Sea) while 21 specimens of the widespread ruffe, G. cernua were sampled at Brateş fish farm pond (Galați County), which has a Danube River water supply. The random sampling of studied fish specimens occurred in 2015, between June and October. Specimen collection was performed using hook - and - line method.

All collected specimens were fixed in $10 \%$ formalin at the site of collection and transported to the laboratory.

A CANON A590 IS camera is used to take digital pictures of the left side of each fish individual. 
Bănărescu (1964) and Holčík and Hensel (1974) describe a total of 18 morphometric measurements (Fig. 1) taken. They are as follows:

- $\quad \mathrm{SL}$ - standard length (mm);

- BODYDEPTH - maximum body height;

- CAUDPEDDEPTH - minimum height of caudal peduncle;

- PREDORSDIST - predorsal distance;

- CAUDPDLENGTH - length of caudal peduncle;

- PREVENTDIST - pre-ventral distance;

- $\quad$ PREANALDIST - pre-anal distance;

- PVDIST - distance between pectoral and ventral fin insertions;

- VADIST - distance between ventral and anal fin insertions;

- DLENGTH - length of dorsal fin base;

- ALENGTH - length of anal fin base;

- ADEPTH - the distance from the insertion of the anal fin to the end point of the highest ray of anal fin;

- PLENGTH - pectoral fin length;

- VLENTGTH - ventral fin length;

- HEADLENGTH - the distance from the tip of the snout to the ventral base of the largest opercula spine;

- HEADDEPTH - head depth;

- MOUTHLENGTH - preorbital length;

- EYEDIAMETER - eye diameter.

The values express a percentage of the standard length, with the exception of the head depth, mouth length and eye diameter, which count as a percentage of the head length. The mean values of each morphological character, as well as the confidence intervals $(\mathrm{P}<0.05)$, were computed using descriptive statistics function with Systat 10.2.

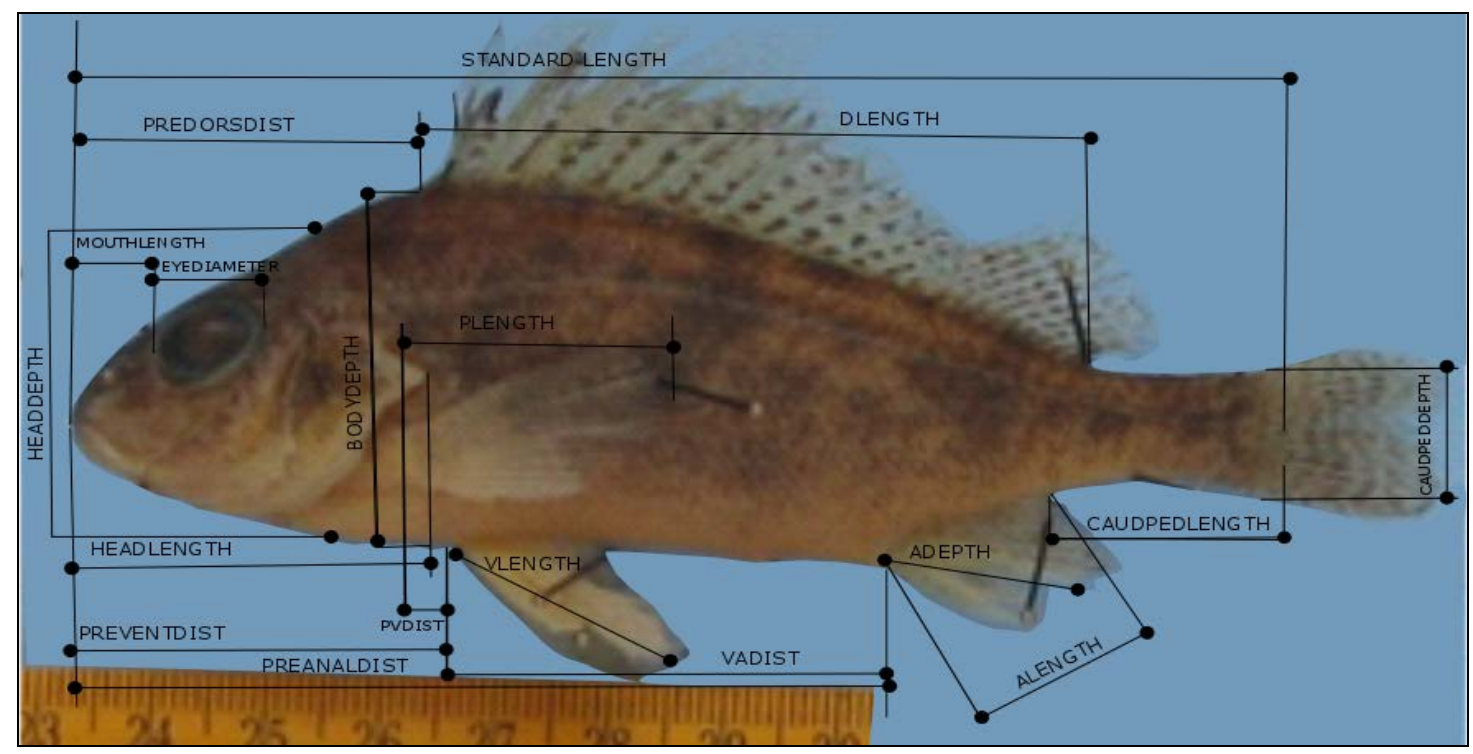

Figure 1: Morphometric characters taken on the ruffe species (picture of Gymnocephalus baloni) studied in the Lower Danube River basin. 
A multivariate approach, including factor analysis (FA) and discriminant analysis (DA), was applied for the morphometric assessment, using Systat 10.2. For DA, a linear discriminant analysis, using a backward stepwise method, based on Mahalanobis distances (developed by Fisher, 1936, in Engelman, 2005), was performed to establish the most relevant morphometric features that together discriminate the samples of G. baloni and G. cernua (Engelman, 2005).

MorfoJ 1.05f applies the geometric methods in this study (Klingenberg, 2011) and 16 landmarks are defined and also recorded as two-dimensional coordinates, using TPS Digitize (Rohlf, 2016a), TPS Relative Warps (Rohlf, 2016b), and TPS Utility (Rohlf, 2016c) software. The landmarks were selected to provide a homogeneous frame of the entire shape. The shape information was extracted by Procrustes superimposition, which removes variation in size, position and orientation from data on landmark coordinates (Klingenberg, 2011).

The multivariate statistical analysis of body shape uses coordinates of the superimposed landmarks, and includes principal components analysis (PCA), discriminant analysis (DA), and canonical variate analysis (CVA) in order to address the main question: the current variability of the ruffe species populations from the Lower Danube River basin, around Mm80 area.

The geometric, statistical analysis provides necessary information regarding the fish body shape variation between groups. The interspecific study used the 49 ruffe individuals.

The statistical test is made using the MorphoJ software. After landmark data importing and shape information extracting with Procrustes superimposition (Klingenberg, 2011), several techniques of multivariate tests data were used to analyse the substantiating patterns in the groups and among the variables. The PCA and CVA statistic tools allow the examination of the relationship among variables and cases in a single block of data (Heyman and Noble, 1989).

\section{RESULTS AND DISCUSSION}

The morphological characters (Fig. 1) obtained through body measurements of the three ruffe species, as well as the confidence intervals with $95 \%$ confidence level, are listed in table 1.

The standard body length of $G$. baloni individuals ranged between $61.72-107.71 \mathrm{~mm}$ (minimum - maximum values), the standard body length of $G$. cernua was $87.74-110 \mathrm{~mm}$, and this of G. schraetser was 161.99-186.46 mm.

Discrimination between G. baloni and its more widespread congener G. cernua assessed by the univariate analysis indicated an overlapping of the ranges in some morphometric characters, except the body depth, length of the caudal peduncle, ventral-anal fin distance, length of dorsal base and eye diameter, which is consistent with references (Holčík and Hensel, 1974).

The received results were compared to those published by other authors (Tabs. 2-4), with several studies made in recent years (Bănărescu, 1964; Holčík and Hensel, 1974; Specziár and Vida, 1995; Kottelat and Freyhof, 2007; Geiger and Schliewen, 2010; Stepien and Haponski, 2015; Tsyba and Kokodiy, 2017). 
Table 1: Main morphological characters of the three ruffe species Gymnocephalus baloni, ( $\mathrm{n}=12$ specimens) Gymnocephalus cernua ( $\mathrm{n}=21$ specimens), and Gymnocephalus schraetser ( $\mathrm{n}=16$ specimens) studied in the Lower Danube River basin (confidence intervals, $\mathrm{P}<0.05 ; \%$ of the standard length except the head depth, mouth length and eye diameter that are expressed as \% of head length). Explanations of morphological characters are in the material and methods.

\begin{tabular}{|l|l|c|c|c|}
\hline \multirow{2}{*}{} & \multicolumn{2}{|c|}{$\begin{array}{c}\text { Morphological } \\
\text { character }\end{array}$} & \multicolumn{3}{|c|}{$\begin{array}{c}\text { Value of morphological character } \\
\text { (Confidence interval, in \% of standard length, } \\
\text { Lhich is in mm unit) }\end{array}$} \\
\cline { 3 - 5 } & \multicolumn{2}{|c|}{$\begin{array}{c}\text { Gymnocephalus } \\
\text { baloni }\end{array}$} & $\begin{array}{c}\text { Gymnocephalus } \\
\text { cernua }\end{array}$ & $\begin{array}{c}\text { Gymnocephalus } \\
\text { schraetser }\end{array}$ \\
\hline 1. & SL & $(82.4-101.8)$ & $(94.3-100.5)$ & $161.99-186.46$ \\
\hline 2. & BODYDEPTH & $27.6-29.1$ & $25.7-26.4$ & $22.12-23.17$ \\
\hline 3. & CAUDPEDDEPTH & $8.5-9.3$ & $8.6-9.1$ & $6.78-7.16$ \\
\hline 4. & PREDORSDIST & $29.5-31.9$ & $29.7-30.9$ & $26.81-28.66$ \\
\hline 5. & CAUDPDLENGTH & $19.8-21.8$ & $21.1-22.4$ & $18.94-20.82$ \\
\hline 6. & PREVENTDIST & $32.1-34.1$ & $33.6-34.8$ & $29.08-30.79$ \\
\hline 7. & PREANALDIST & $63.8-66.7$ & $64.3-65.8$ & $66.51-68.84$ \\
\hline 8. & PVDIST & $3.5-4.5$ & $2.8-3.6$ & $3.50-4.73$ \\
\hline 9. & VADIST & $31.1-34.9$ & $30.6-31.8$ & $36.48-39.21$ \\
\hline 10. & DLENGTH & $54.1-56.8$ & $53.0-54.7$ & $55.83-57.97$ \\
\hline 11. & ALENGTH & $14.4-17.0$ & $13.9-15.2$ & $12.06-13.60$ \\
\hline 12. & ADEPTH & $19.2-23.2$ & $19.5-20.8$ & $17.53-19.77$ \\
\hline 13. & PLENGTH & $21.4-24.0$ & $22.2-23.6$ & $17.76-18.66$ \\
\hline 14. & VLENTGTH & $21.1-22.6$ & $20.6-22.2$ & $18.50-19.50$ \\
\hline 15. & HEADLENGTH & $30.4-32.7$ & $31.5-32.6$ & $25.39-28.43$ \\
\hline 16. & HEADDEPTH & $75.7-79.9$ & $72.3-74.8$ & $68.88-79.50$ \\
\hline 17. & MOUTHLENGTH & $26.4-29.8$ & $27.5-29.6$ & $37.51-41.49$ \\
\hline 18. & EYEDIAMETER & $29.2-31.9$ & $27.3-29.2$ & $24.46-28.73$ \\
\hline
\end{tabular}

Our results were consistent with some authors (Tab. 2), however showed differences in some of the morphological features when compared with other scientifical researches (the body depth, predorsal distance, caudal peduncle length, preventral distance, and preanal distance). 
Table 2: Main morphological characters of Gymnocephalus baloni specified by various references (\% of the standard length except for the head depth, mouth length and eye diameter). Explanations of morphological characters are in the material and methods.

\begin{tabular}{|c|c|c|c|c|c|c|c|c|c|c|}
\hline 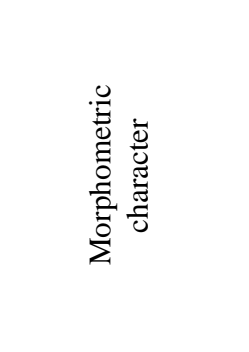 & 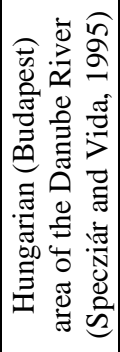 & 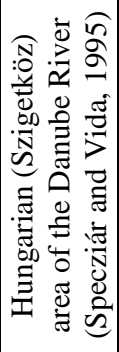 & 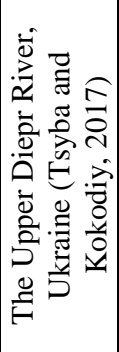 & 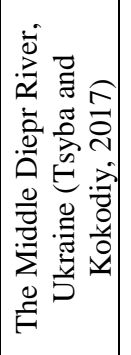 & 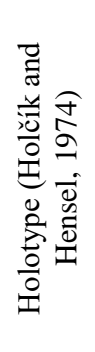 & 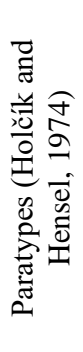 & 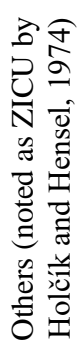 & 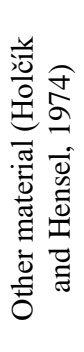 & 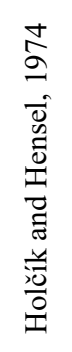 & 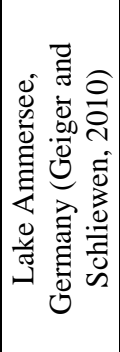 \\
\hline STANDLENGTH & 78.8 & 97.5 & 96.4 & 93.2 & 107.3 & 96.5 & 112.6 & 93.7 & $\begin{array}{c}\text { no } \\
\text { data }\end{array}$ & 101.7 \\
\hline BODYDEPTH & 29.9 & 31.2 & 31.6 & 31.4 & 31.6 & 31.2 & 35.2 & 31.0 & 30.8 & 32.1 \\
\hline CAUDPEDDEPTH & 8.1 & 8.2 & 8.4 & 8.3 & 8.4 & 8.7 & 10.4 & 8.7 & 8.6 & 8.9 \\
\hline PREDORSSDIST & 32.4 & 31.4 & 37.2 & 37.1 & 35.3 & 33.8 & 40.0 & 34.4 & 34.1 & 35.4 \\
\hline CAUDPDLENGTH & 21.2 & 20.2 & 21.0 & 16.8 & 18.6 & 19.7 & 22.4 & 19.9 & 19.8 & 17.9 \\
\hline PREVENTDIST & 36.1 & 36.7 & 35.3 & 36.4 & 39.4 & 39.1 & 41.7 & 38.8 & 38.7 & 37.0 \\
\hline PREANALDIST & 66.8 & 69.1 & 68.0 & 69.7 & 70.3 & 70.3 & 77.8 & 70.2 & $\begin{array}{c}\text { no } \\
\text { data }\end{array}$ & $\begin{array}{c}\text { no } \\
\text { data }\end{array}$ \\
\hline PVDIST & 3.5 & 4.5 & 6.0 & 4.9 & 12.6 & 12.3 & 12.9 & 12.1 & 12.0 & $\begin{array}{l}\text { no } \\
\text { data }\end{array}$ \\
\hline VADIST & 30.7 & 32.5 & 32.3 & 34.7 & 32.7 & 34.7 & 38.6 & 34.3 & $\begin{array}{c}\text { no } \\
\text { data }\end{array}$ & $\begin{array}{c}\text { no } \\
\text { data }\end{array}$ \\
\hline DLENGTH & 50.6 & 52.4 & 56.75 & 56.9 & 58.2 & 56.4 & 64.8 & 56.7 & 56.3 & $\begin{array}{c}\text { no } \\
\text { data }\end{array}$ \\
\hline ALENGTH & 11.9 & 10.7 & 14.8 & 14.8 & 13.9 & 14.4 & 18.0 & 14.5 & 14.4 & 14.6 \\
\hline ADEPTH & 16.7 & 14.7 & 17.2 & 16.6 & $\begin{array}{c}\text { no } \\
\text { data }\end{array}$ & $\begin{array}{c}\text { no } \\
\text { data }\end{array}$ & $\begin{array}{c}\text { no } \\
\text { data }\end{array}$ & $\begin{array}{c}\text { no } \\
\text { data }\end{array}$ & $\begin{array}{c}\text { no } \\
\text { data }\end{array}$ & $\begin{array}{c}\text { no } \\
\text { data }\end{array}$ \\
\hline PLENGTH & 21.0 & 20.4 & 22.1 & 21.1 & 20.1 & 19.4 & 23.8 & 19.8 & $\begin{array}{c}\text { no } \\
\text { data }\end{array}$ & 19.7 \\
\hline VLENGTH & 22.4 & 21.4 & 21.8 & 22.9 & 22.3 & 21.4 & 24.8 & 21.9 & $\begin{array}{c}\text { no } \\
\text { data }\end{array}$ & 20.3 \\
\hline HEADLENGTH & 33.0 & 31.5 & 31.8 & 32.2 & 25.6 & 30.1 & 35.1 & 30.5 & $\begin{array}{c}\text { no } \\
\text { data }\end{array}$ & 29.2 \\
\hline HEADDEPTH & 64.2 & 73.0 & 80.2 & 76.1 & 105.1 & 85.7 & 82.1 & 84.9 & $\begin{array}{c}\text { no } \\
\text { data }\end{array}$ & 84.3 \\
\hline MOUTHLENGTH & $\begin{array}{c}\text { no } \\
\text { data }\end{array}$ & 0.0 & 32.4 & 35.2 & 39.8 & 33.5 & 32.2 & 33.1 & $\begin{array}{c}\text { no } \\
\text { data }\end{array}$ & 35.5 \\
\hline EYEDIAMETER & 28.8 & 28.3 & 31.4 & 29.9 & 33.6 & 28.6 & 28.8 & 29.2 & $\begin{array}{c}\text { no } \\
\text { data }\end{array}$ & 31.8 \\
\hline
\end{tabular}

The body depth of G. baloni sampled from the Lower Danube River is smaller than the parameter measured on the holotype (Tab. 2) and smaller than other samples from the Danube River basin showing the specific phenotypic response to lotic habitat and unsteady swimming behaviour of fish. We can mention here the results of Foster et al. (2015), which highlight the general influence of the body shape differences by different habitat types and adaptive phenotypic features in the sympatric species to relative environmental gradients. 
Table 3: Main morphological characters of Gymnocephalus cernua specified by various references (\% of standard length except for the head depth, mouth length and eye diameter). Explanations of morphological characters are in the material and methods.

\begin{tabular}{|c|c|c|c|c|c|c|c|c|c|c|c|c|}
\hline 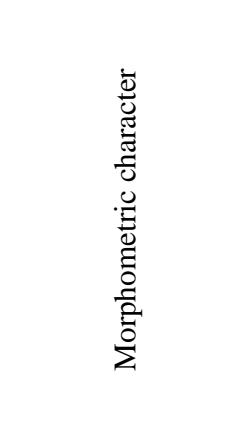 & 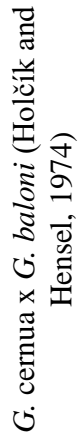 & 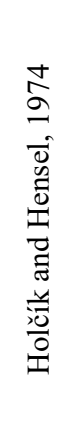 & 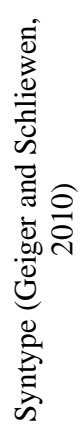 & 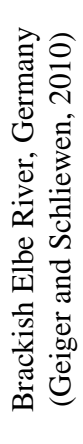 & 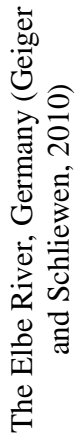 & 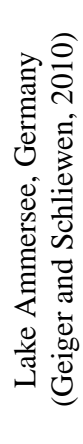 & 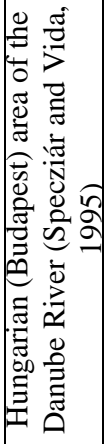 & 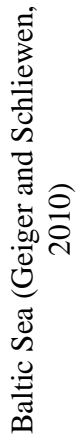 & 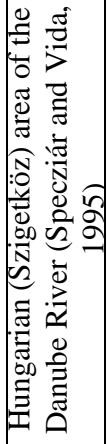 & 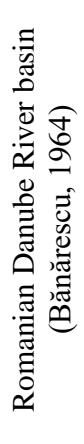 & 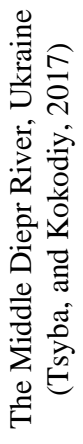 & 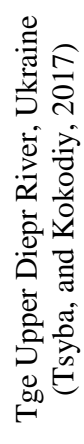 \\
\hline STANDLENGTH & $\begin{array}{c}\text { no } \\
\text { data }\end{array}$ & $\begin{array}{c}\text { no } \\
\text { data }\end{array}$ & 101.1 & 100.3 & 80.5 & 98.9 & 91.9 & 101.3 & 84.9 & 145.0 & 103.8 & 93.3 \\
\hline BODYDEPTH & 30.2 & 25.8 & 35.3 & 27.0 & 26.6 & 27.0 & 25.8 & 23.4 & 27.2 & 29.5 & 25.3 & 27.0 \\
\hline CAUDPEDDEPTH & 8.9 & 8.2 & 10.1 & 9.2 & 9.4 & 8.8 & 8.0 & 7.5 & 8.2 & 8.4 & 7.7 & 7.8 \\
\hline PREDORSSDIST & 34.8 & 32.7 & 36.6 & 38.3 & 36.2 & 35.1 & 30.8 & 34.2 & 31.8 & 34.0 & 35.2 & 35.2 \\
\hline CAUDPDLENGTH & 20.9 & 21.6 & 17.9 & 20.1 & 21.2 & 20.2 & 22.9 & 23.3 & 23.1 & 21.0 & 20.1 & 24.6 \\
\hline PREVENTDIST & 36.6 & 36.4 & 39.1 & 36.8 & 35.0 & 35.2 & 35.0 & 32.2 & 34.4 & $\begin{array}{c}\text { no } \\
\text { data }\end{array}$ & 33.9 & 33.3 \\
\hline PREANALDIST & $\begin{array}{c}\text { no } \\
\text { data }\end{array}$ & $\begin{array}{c}\text { no } \\
\text { data }\end{array}$ & $\begin{array}{c}\text { no } \\
\text { data }\end{array}$ & $\begin{array}{c}\text { no } \\
\text { data }\end{array}$ & $\begin{array}{c}\text { no } \\
\text { data }\end{array}$ & $\begin{array}{c}\text { no } \\
\text { data }\end{array}$ & 67.2 & $\begin{array}{c}\text { no } \\
\text { data }\end{array}$ & 67.5 & $\begin{array}{c}\text { no } \\
\text { data }\end{array}$ & 67.2 & 65.3 \\
\hline PVDIST & 11.5 & 9.7 & $\begin{array}{c}\text { no } \\
\text { data }\end{array}$ & $\begin{array}{c}\text { no } \\
\text { data }\end{array}$ & $\begin{array}{c}\text { no } \\
\text { data }\end{array}$ & $\begin{array}{c}\text { no } \\
\text { data }\end{array}$ & 3.2 & $\begin{array}{c}\text { no } \\
\text { data }\end{array}$ & 2.6 & $\begin{array}{c}\text { no } \\
\text { data }\end{array}$ & 4.3 & 5.9 \\
\hline VADIST & $\begin{array}{c}\text { no } \\
\text { data }\end{array}$ & $\begin{array}{c}\text { no } \\
\text { data }\end{array}$ & $\begin{array}{c}\text { no } \\
\text { data }\end{array}$ & $\begin{array}{c}\text { no } \\
\text { data }\end{array}$ & $\begin{array}{c}\text { no } \\
\text { data }\end{array}$ & $\begin{array}{c}\text { no } \\
\text { data }\end{array}$ & 32.3 & $\begin{array}{c}\text { no } \\
\text { data }\end{array}$ & 33.2 & $\begin{array}{c}\text { no } \\
\text { data }\end{array}$ & 34.6 & 32.1 \\
\hline DLENGTH & 5.7 & 53.8 & $\begin{array}{c}\text { no } \\
\text { data }\end{array}$ & $\begin{array}{c}\text { no } \\
\text { data }\end{array}$ & $\begin{array}{c}\text { no } \\
\text { data }\end{array}$ & $\begin{array}{c}\text { no } \\
\text { data }\end{array}$ & 50.8 & $\begin{array}{c}\text { no } \\
\text { data }\end{array}$ & 50.3 & $\begin{array}{c}\text { no } \\
\text { data }\end{array}$ & 53.5 & 53.2 \\
\hline ALENGTH & 13.4 & 12.5 & 15.0 & 14.7 & 13.0 & 12.4 & 9.9 & 11.4 & 9.3 & $\begin{array}{c}\text { no } \\
\text { data }\end{array}$ & 12.3 & 11.9 \\
\hline ADEPTH & $\begin{array}{c}\text { no } \\
\text { data }\end{array}$ & $\begin{array}{c}\text { no } \\
\text { data }\end{array}$ & $\begin{array}{c}\text { no } \\
\text { data }\end{array}$ & $\begin{array}{c}\text { no } \\
\text { data } \\
\end{array}$ & $\begin{array}{c}\text { no } \\
\text { data }\end{array}$ & $\begin{array}{c}\text { no } \\
\text { data }\end{array}$ & 16.7 & $\begin{array}{c}\text { no } \\
\text { data }\end{array}$ & 15.7 & $\begin{array}{c}\text { no } \\
\text { data }\end{array}$ & 14.7 & 14.8 \\
\hline PLENGTH & $\begin{array}{c}\text { no } \\
\text { data }\end{array}$ & $\begin{array}{c}\text { no } \\
\text { data }\end{array}$ & 21.1 & 23.6 & 20.6 & 20.1 & 20.5 & 18.5 & 22.2 & 22.0 & 20.4 & 20.8 \\
\hline VLENGTH & $\begin{array}{c}\text { no } \\
\text { data }\end{array}$ & $\begin{array}{c}\text { no } \\
\text { data }\end{array}$ & 22.0 & 22.5 & 20.4 & 19.8 & 21.6 & 18.6 & 22.6 & 21.8 & 21.3 & 19.7 \\
\hline HEADLENGTH & $\begin{array}{c}\text { no } \\
\text { data }\end{array}$ & $\begin{array}{c}\text { no } \\
\text { data }\end{array}$ & 30.6 & 33.2 & 31.3 & 29.5 & 31.8 & 28.7 & 31.7 & 31.3 & 30.9 & 30.5 \\
\hline HEADDEPTH & $\begin{array}{c}\text { no } \\
\text { data }\end{array}$ & $\begin{array}{c}\text { no } \\
\text { data }\end{array}$ & 86.3 & 71.4 & 72.2 & 75.9 & 59.7 & 69.3 & 64.0 & $\begin{array}{c}\text { no } \\
\text { data }\end{array}$ & 66.6 & 71.0 \\
\hline MOUTHLENGTH & $\begin{array}{c}\text { no } \\
\text { data }\end{array}$ & $\begin{array}{c}\text { no } \\
\text { data }\end{array}$ & 33.9 & 36.9 & 35.6 & 37.1 & 0.0 & 36.6 & 0.0 & 32.8 & 34.0 & 30.2 \\
\hline EYEDIAMETER & $\begin{array}{c}\text { no } \\
\text { data }\end{array}$ & $\begin{array}{c}\text { no } \\
\text { data }\end{array}$ & 29.9 & 28.1 & 31.1 & 29.6 & 26.6 & 31.6 & 29.6 & 27.3 & 27.6 & 29.7 \\
\hline
\end{tabular}


Table 4: Main morphological characters of Gymnocephalus schraetser from the Lower Danube River basin (Bănărescu, 1964). Explanations of morphological characters are in the material and methods.

\begin{tabular}{|c|c|}
\hline $\begin{array}{l}\text { Morphometric } \\
\text { character }\end{array}$ & $\begin{array}{l}\text { Value (\% of standard length except the head } \\
\text { depth, mouth length and eye diameter) }\end{array}$ \\
\hline STANDLENGTH & 170.0 \\
\hline BODYDEPTH & 21.8 \\
\hline CAUDPEDDEPTH & 6.8 \\
\hline PREDORSSDIST & 32.8 \\
\hline CAUDPDLENGTH & 20.5 \\
\hline PREVENTDIST & no data \\
\hline PREANALDIST & no data \\
\hline PVDIST & no data \\
\hline VADIST & no data \\
\hline DLENGTH & no data \\
\hline ALENGTH & no data \\
\hline ADEPTH & no data \\
\hline PLENGTH & 18.6 \\
\hline VLENGTH & 19.8 \\
\hline HEADLENGTH & 31.5 \\
\hline HEADDEPTH & no data \\
\hline MOUTHLENGTH & 46.0 \\
\hline EYEDIAMETER & 22.7 \\
\hline
\end{tabular}

In the same way, as an adaptive answer of fish morphology to water flow regime and other ecological parameters, we recorded smaller values in Danube ruffe body characters than the holotype description for some biometric characters such as predorsal distance, preventral distance, preanal distance. These differences were not recorded by comparing measurements of the fish population sampled from Hungarian Danube River section (Tab. 2) and also to populations in the upper and middle basin of the Dnieper/Nistru River (Tab. 2).

A suggestive image of the morphological variation and relationship among species from the genus Gymnocephalus sampled from the Lower Danube River (Mm80), compared with results from the measurements of fish from the various Eurasian rivers obtained by different authors (Tabs. 2-4) was assessed by a hierarchical clustering single linkage method. The dendrogram of figure 2 displays the output of the hierarchical clustering, which represent the trees that put in evidence, the similarity, and dissimilarity between the results. 
The cluster based on our results reveales a more advanced degree of morphological similarity of fish with data referring to fish sampled from the upper and middle Dniepr/Nistru Basin (Tabs. 2-4) and the Hungarian stretch of Danube River (Tabs. 2-4). The distances between our results and the clusters of Danube ruffe holotype/paratype (Tab. 2) and widespread ruffe paratype (Tab. 3) are bigger (Fig. 2).

The dendrogram sustains the identified dissimilarity between G. schraetser (according to data described by Bănărescu, 1964 on 26 specimens from Danube, Timiş, Bega, Mureş rivers, Romania, Tab. 4) and the other two ruffe species (2.20 Euclidean distance).

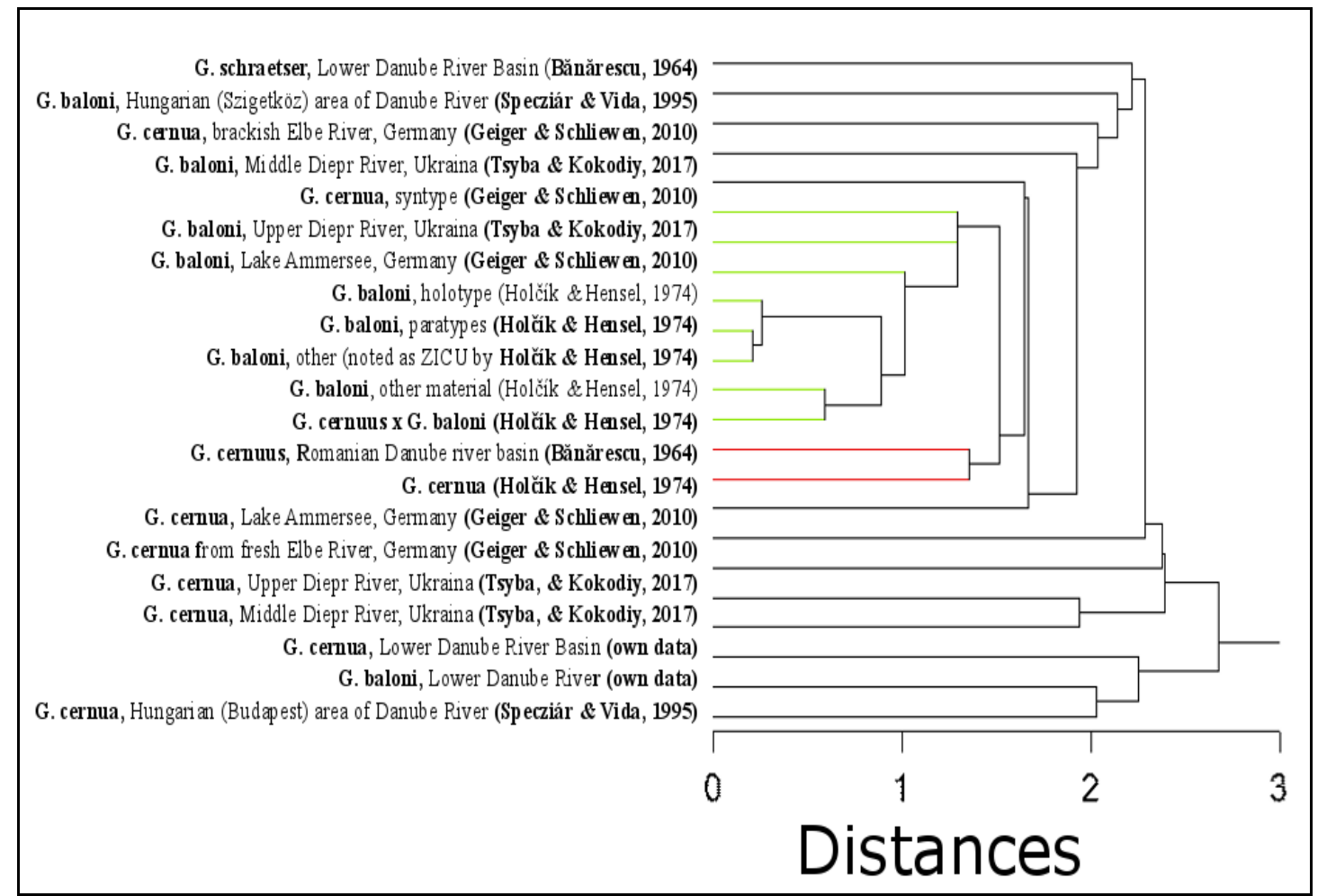

Figure 2: Dendrogram of the hierarchical clustering analysis based on morphometric data.

The algorithm used was the single linkage clustering (nearest neighbour) with the Euclidean distance as a similarity measure.

Aco-variance matrix of the Procrustes coordinates of each sample is used to perform the PCA, and the first two components summarised $67.166 \%$, which represent more than the half of total variance.

The scatter plot of PC scores from figure 3 displays the patterns in the relations between observations. PC1 separated G. schraetser (blue circle) from the other two studied species of interest while PC2 separated G. baloni (red circle) from G. cernua (green circle) (Fig. 3). 


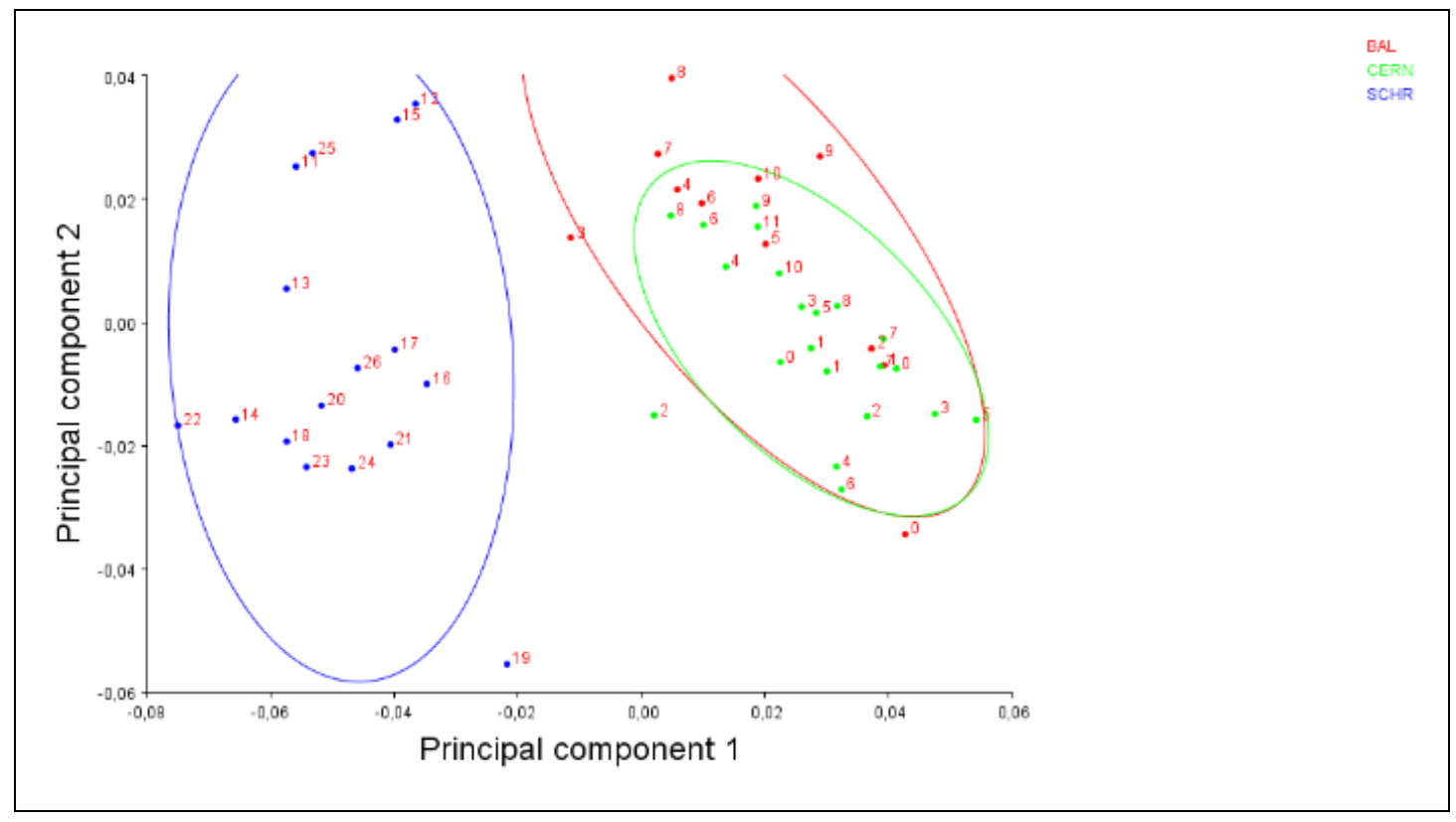

Figure 3: PC scores and the disposition of specimens in the morphospace of the multivariate analysis. The coordinates of PCA plot are grouped by equal frequency confidence ellipses ( $\mathrm{P}=0.9$ ), which separate $G$. schraetser (blue circle) G. baloni (red circle), and G. cernua (green circle).

In order to have an optimised representation of the differences among groups, we performed a canonical variate analysis (CVA) that allows a different type of coordination analysis, which improves the separation of the specified groups (species/ecotypes; Klingenberg, 2011).

Figure 4 represents the shape changes associated with the canonical variate in the species from the genus Gymnocephalus in the Lower Danube. The scale factor for canonical variate (CV) shape changes is a unit of Mahalanobis distance of within-group shape changes (Klingenberg, 2011). We observed the variation in the pairs of fin insertions, eye diameter, head length and body depth, which is in connection with the elongation degree of body shape.

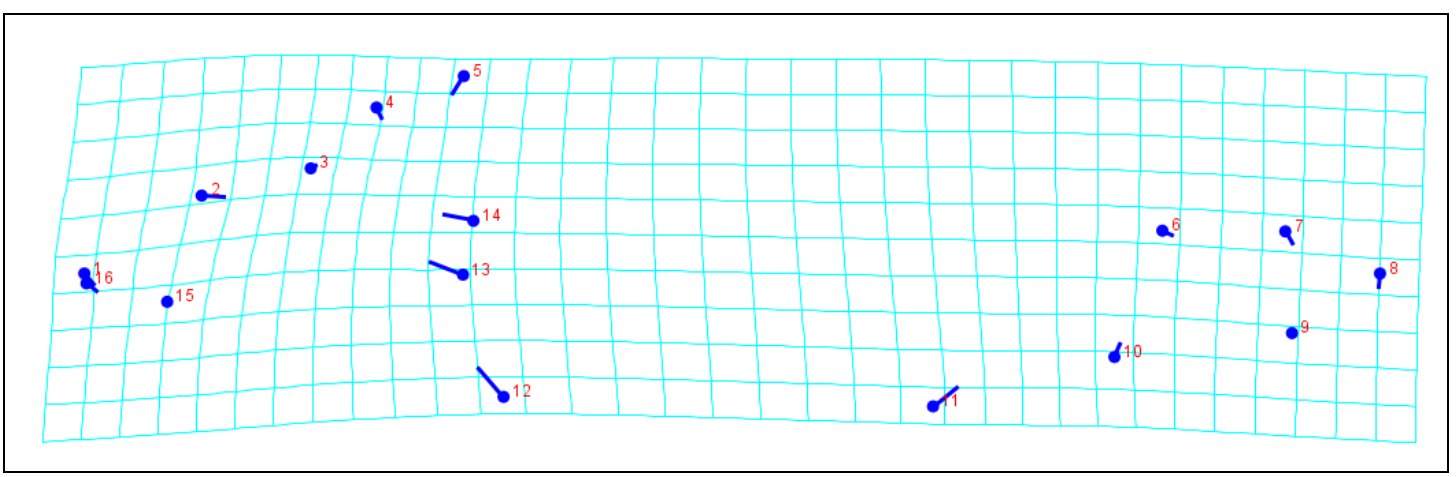

Figure 4: Transformation grid illustrating the shape variation associated with canonical variates (CV) in the ruffe species from the Lower Danube River. The scale for CV shape changes is in units of Mahalanobis distance. 
The CVA generated the matrices of farthest Mahalanobis distances between the groups of $G$. baloni and $G$. schraetser (18.3364) and the smallest distances between $G$. baloni and G. cernua (8.7875). The P-values from permutation tests (10.000 permutation rounds) for Mahalanobis distances among the groups were statistically significant $(\mathrm{P}<0.05)$.

The CVA chart represents the scatter plot of the canonical variate scores, displaying the species differentiation. The figure 5 allows the description of the relationship among the specimens of the groups by plotting the sample centroids of $90 \%$ confidence ellipses of the first two canonical variates CV1 and CV2.

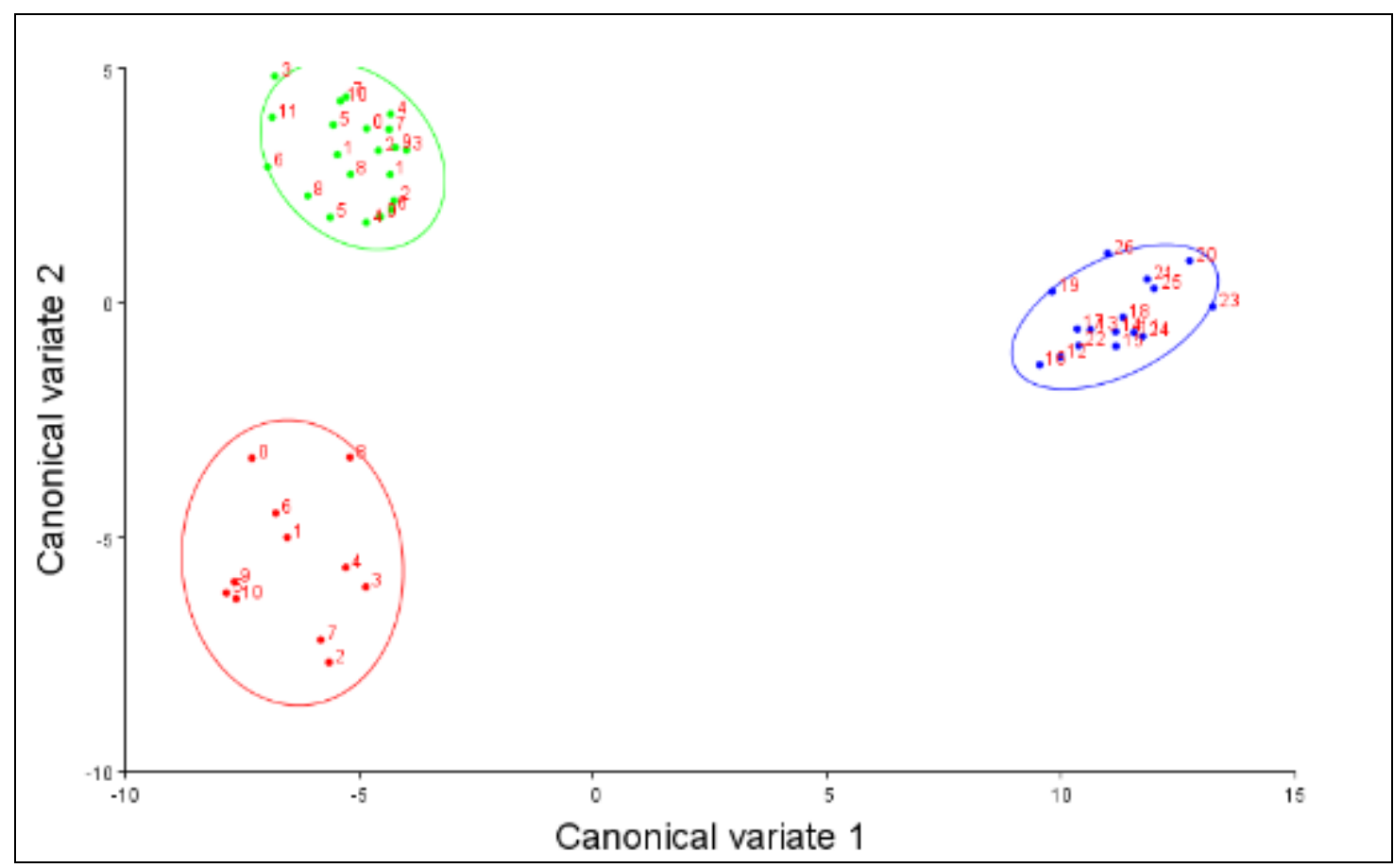

Figure 5: Transformation grid illustrating the shape variation

associated with canonical variates (CV) in the ruffe species from the Lower Danube River.

The scale for CV shape changes is in units of Mahalanobis distance.

The statistical analysis of morphometric data is for G. baloni and G. cernua. The objective is in order to point out the phenotypic characters for reliable discrimination between these two species.

The performed factor analysis reveales the first three principal components sum up $59.267 \%$, which means that the first three factors explain more than a half of the variance of all body variables (Fig. 6). Based on the variables with high loadings, it may be seen that PC1 includes the significant, positive contribution of the dorsal fin length, eye diameter, head depth and the negative contribution of the head length, mouth length, preanal, predorsal, preventral and preorbital distances (Fig. 6a). In the same way, PC2 indicates the positive contribution of characters as the pectoral-ventral fin distance, ventral-anal fin distance, and preanal distance, and the negative contribution of the caudal peduncle length (Fig. 6b). PC3 only reveales the positive contribution of the body depth, ventral length, anal fin length, and anal fin depth. 


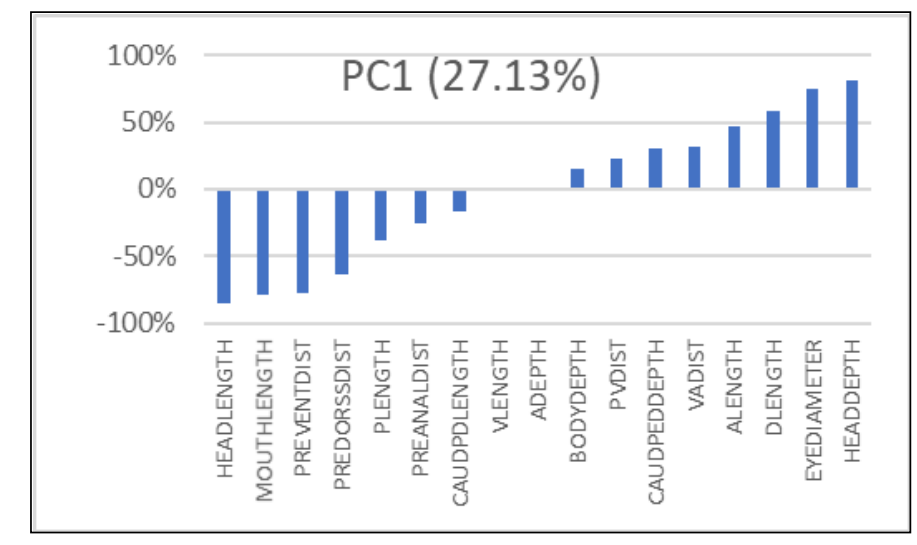

a)

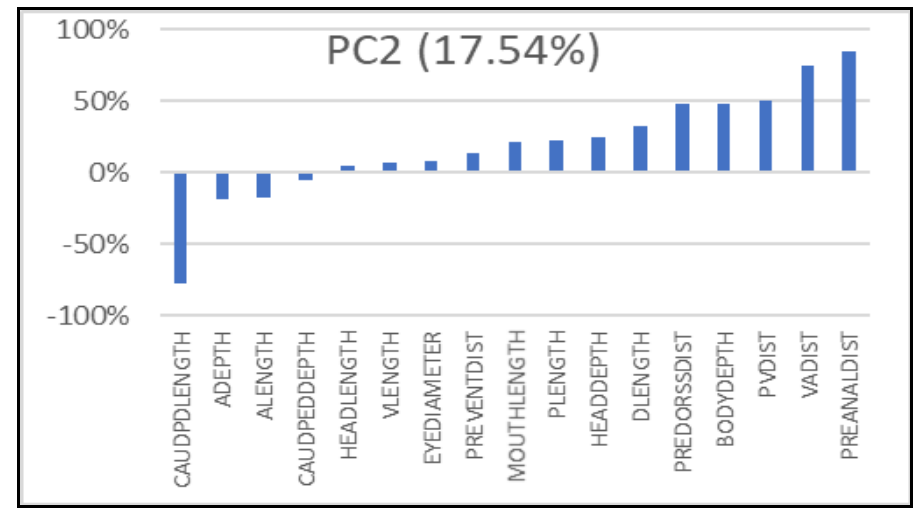

b)

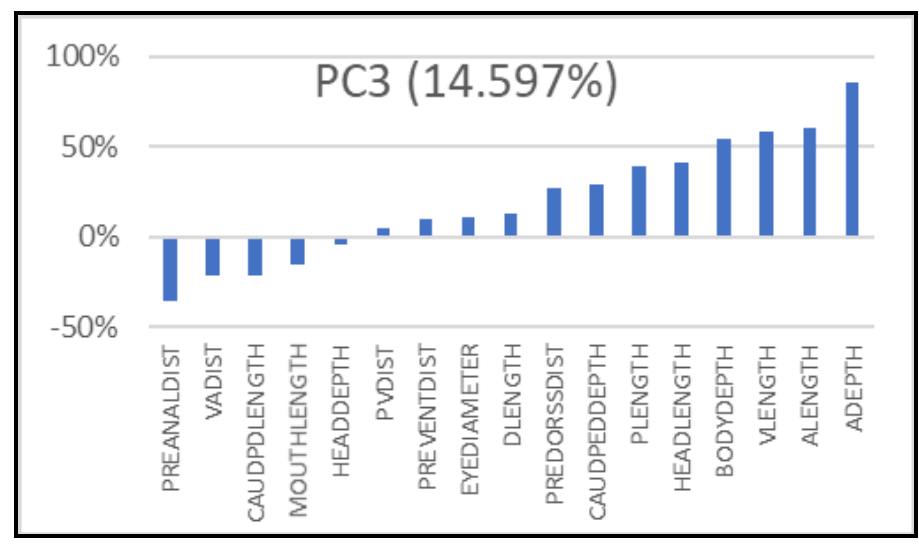

c)

Figure 6a-c: Variables loaded in the factor analysis of the morphometric measurement values of Gymnocephalus baloni and Gymnocephalus cernua $(\mathrm{n}=32)$; the full names of variables are in material and methods. 
We tested the differences between samples with $\mathrm{T}$ test. Table 5 notes the significant differences in the main characters of fish from the two groups $(\mathrm{P}<0.05)$ as a character " $\mathrm{S}$ ".

Table 5: Mean values of variables (\%) in Gymnocephalus baloni and Gymnocephalus cernua. Explanations of morphological characters are in the material and methods. $S$ - significant differences.

\begin{tabular}{|l|l|l|l|}
\hline \multicolumn{1}{|c|}{ No. } & \multicolumn{1}{c|}{$\begin{array}{c}\text { Morphological } \\
\text { character }\end{array}$} & \multicolumn{1}{c|}{$\begin{array}{c}\text { G. baloni } \\
\text { mean (\%) }\end{array}$} & \multicolumn{1}{c|}{$\begin{array}{c}\text { G. cernua } \\
\text { mean (\%) }\end{array}$} \\
\hline 1. & BODYDEPTH & 28.327 & 26.043 \\
\hline 2. & CAUDPEDDEPTH & 8.945 & 8.819 \\
\hline 3. & PREDORSSDIST & 30.691 & 30.305 \\
\hline 4. & CAUDPDLENGTH & 20.827 & 21.767 \\
\hline 5. & PREVENTDIST & 33.118 & 34.181 \\
\hline 6. & PREANALDIST & 65.282 & 65.076 \\
\hline 7. & PVDIST & 4.000 & 3.205 \\
\hline 8. & VADIST & 32.982 & 31.210 \\
\hline 9. & DLENGTH & 55.473 & 53.833 \\
\hline 10. & ALENGTH & 15.664 & 14.538 \\
\hline 11. & ADEPTH & 21.191 & 20.171 \\
\hline 12. & PLENGTH & 22.673 & 22.914 \\
\hline 13. & VLENGTH & 21.845 & 21.362 \\
\hline 14. & HEADLENGTH & 31.555 & 32.048 \\
\hline 15. & HEADDEPTH & 77.800 & 73.581 \\
\hline 16. & MOUTHLENGTH & 28.118 & 28.552 \\
\hline 17. & EYEDIAMETER & 30.518 & 28.248 \\
\hline
\end{tabular}

Having in view the similarity between G. baloni and G. cernua, a multivariate test for a morphometric comparison between the collected samples from the Danube River population (sea mile 80 from Black Sea) is used to complete the data regarding the morphological variation in the ruffe species and assess its plasticity.

A linear discriminant analysis using a backward stepwise method allows establishment of the most relevant morphometric features of $G$. baloni and G. cernua that together discriminate between the two samples of the fish species.

The between groups F-matrix and analysis of the disparities between the groups and all the variables (Engelman, 2005) reveals the correct classification of the ruffe samples with the farthest distance between G. baloni and G. cernua (6.754).

The reliability assessment of the discriminant analysis of morphometric characters of fish from the two species, computed by Wilks' lambda statistical index (Engelman, 2005) proved a significant discrimination with an advanced degree of confidence (Wilks' lambda = 0.1514 , prob $=0.00008, \mathrm{P}<0.05$ ).

The F-to-remove statistics (Engelman, 2005) from table 6 allows us to conclude that 10 of the morphological characters are the most useful together for discriminating among the species (the head features, body depth, the distances between pectoral, ventral and respectively anal fins, and the lengths of fin bases). 
Table 6: F-to-remove statistics of the main morphometric characters assessed by the backward stepwise method for discrimination among the ruffe species: Gymnocephalus baloni and Gymnocephalus cernua. Explanations of morphological characters are in the material and methods.

\begin{tabular}{|l|c|c|}
\hline Variable & F-to-remove & Tolerance \\
\hline BODYDEPTH & 22.69 & 0.200 \\
\hline PREDORSDIST & 6.11 & 0.219 \\
\hline PREANALDIST & 14.09 & 0.115 \\
\hline PVDIST & 18.43 & 0.255 \\
\hline VADIST & 8.27 & 0.332 \\
\hline ALENGTH & 4.15 & 0.334 \\
\hline PLENGTH & 5.56 & 0.356 \\
\hline VLENGTH & 3.65 & 0.560 \\
\hline HEADLENGTH & 6.09 & 0.057 \\
\hline HEADDEPTH & 4.16 & 0.09 \\
\hline
\end{tabular}

The classification matrix showed a high percentage of classification validation in all cases (100\% correct classification).

Jackknife a form of cross-validation using a method of leaving out one case confirms the correct classification of the two sympatric ruffe species. The relative lower percent in the Jackknife panel (73\% correct) could be attributed to the high number of predictors and small size of the ruffe samples.

The canonical scores plot generate the graphic with the confidence ellipse $(\mathrm{P}<0.05)$ of each group, which is centred on the centroid of each group (Fig. 7). Based on the body measurement values, the groups are distinct.

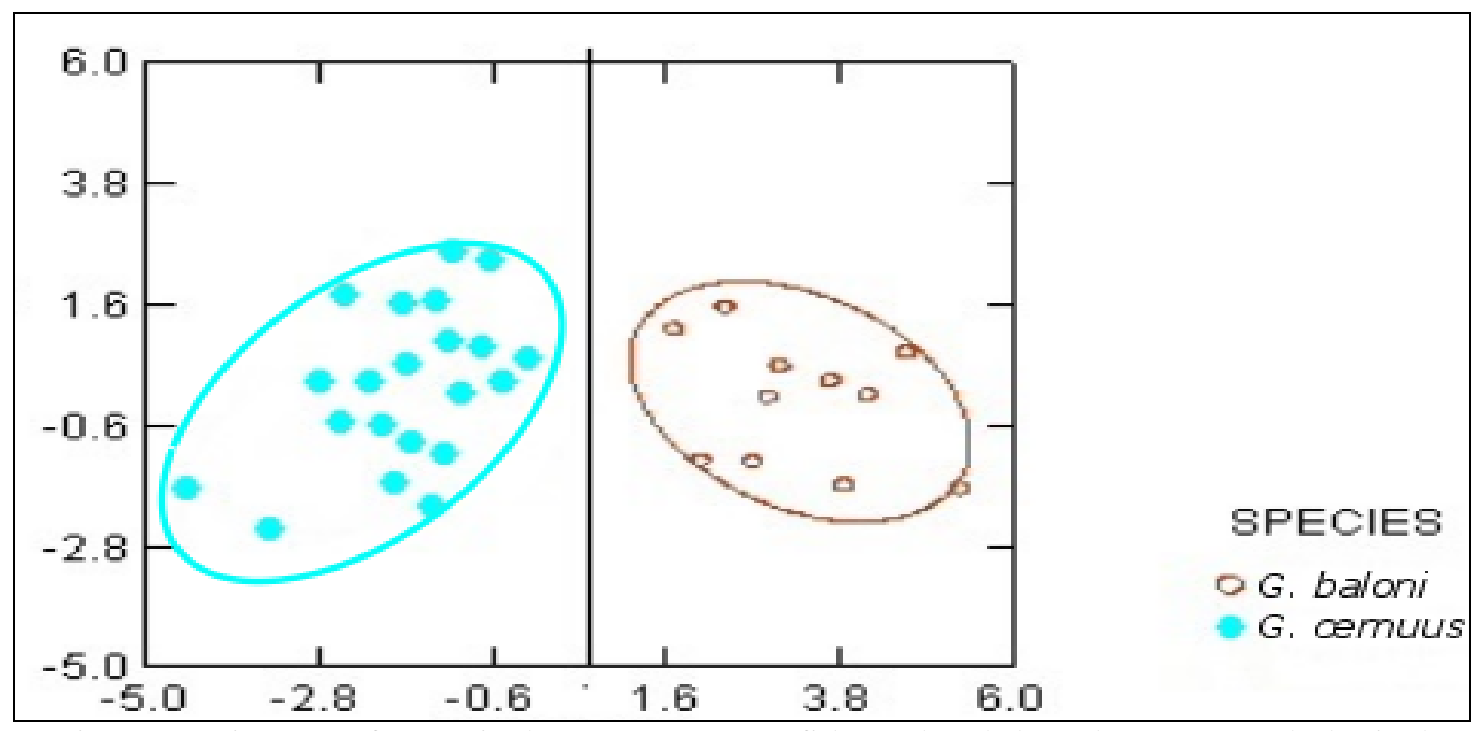

Figure 7: Diagram of canonical scores (95\% confidence level) based on 18 morphological characters (Tab. 1) for Gymnocephalus cernua (blue circle) and Gymnocephalus baloni (brown circle) of the samples on which a discriminant analysis was applied (95\% confidence ellipses are drawn around group centroids, and the variance proportions represent each canonical function as indicated). 


\section{CONCLUSIONS}

The current paper completes the information regarding the body plasticity of the Danube ruffe (Gymnocephalus baloni) and its relationship with the other two species of the genus Gymnocephalus from the Lower Danube River.

We revealed a closer relationship between the population of Gymnocephalus baloni from the Lower Danube River and those from the middle stretch of the Danube River and the populations identified in rivers from the eastern part of Europe.

We found a closer phylogenetic relationship between the sister taxa G. cernua and Gymnocephalus baloni and a distance from Gymnocephalus schraetser, results that are consistent with some of previous studies.

Ten morphological characters are the most useful together for discriminating among the ruffe species from the Lower Danube River.

We also found a slightly less hydrodynamic body of Gymnocephalus baloni from the Lower Danube River comparing with the description of the holotype, which could be in connection with the phenotypic response of fish to environment. 


\section{ACKNOWLEDGEMENTS}

The authors gratefully acknowledge the following individuals for their support: the anonymous reviewers, Dr. Curtean-Bănăduc A. and Dr. Bănăduc D., the editors of Transylvanian Review of Systematical and Ecological Research for the valuable, critical and helpful comments on the manuscript, and the fishers operating in Lower Danube River for cooperation and providing the fish samples. 


\section{REFERENCES}

1. Antipa G., 1905 - Die Störe und ihre Wanderungen in den europäischen Gewässern, mit besonderer Berücksichtigung der Störe der Donau und des Schwarzen Meeres, Internationalen Fisherei Kongress, Wien. (in German)

2. Antipa G., 1909 - Fauna ihtiologică a României, Publicaţiile Fondului Adamachi, 294. (in Romanian)

3. Antipa G., 1915 - Wissenschaftliche und Wirtschaftliche Probleme des Donaudeltas, Göbl, Bukarest 1909, 88. (in German)

4. Antipa G., 1933 - Pescăriile și regiunea inundabilă a Dunării în cadrul economiei naționale și mondiale, Monitorul Oficial și Imprimeriile Statului, Imprimeria Centrală, Bucureşti, 312, (in Romanian)

5. Antipa G., 1934 - Les sturions de la Mer Noir, 16, 67-83. (in French)

6. Bănăduc D. S., 2009 - Lower Timiş River (Banat, Romania) - Special area for conservation (Natura 2000 site) for Gobio albipinnatus Lukasch, 1933, AACL Bioflux, 2, 2, 147-151.

7. Bănăduc D., Curtean-Bănăduc A., Lenhardt M. and Guti G., 2014 - "Porţile de Fier/Iron Gates" Gorges area (Danube) fish fauna, Transylvanian Review of Systematical and Ecological Research, 16 - special issue, The "Iron Gates” Natural Park, Edit. Universităţii „Lucian Blaga” din Sibiu, ISSN 1841-7051, 171-196.

8. Bănăduc D., Rey S., Trichkova T., Lenhardt M. and Curtean-Bănăduc A., 2016 - The Lower Danube River - Danube Delta - North West Black Sea: A pivotal area of major interest for the past, present and future of its fish fauna - A short review, Science of the Total Environment, 545-546, 137-151, DOI: 10.1016/j.scitotenv.2015.12.058, ISSN 0048-9607, eISSN 1879-1026, March 2016.

9. Bănărescu M. P., 1964 - Pisces-Osteichthyes, Fauna R. P. R., XIII, Edit. Acad. R. S. R., Bucharest, 959. (in Romanian)

10. Bănărescu P. M., 2005 - Pisces (Pești) in Cartea Roșie a vertebratelor din România, Botnariuc N. and Tatole V. (eds), Muzeul Naţional de Istorie Naturală „Grigore Antipa”, Bucureşti, 215 254. (in Romanian)

11. Bănărescu P. M. and Bănăduc D., 2007 - Habitats directive (92/43/EEC). Fish species (Osteichthyes) on the Romanian territory, Acta Ichtiologica Romanica, II, 43-78.

12. Bulat D., 2017 - Ihtiofauna Republicii Moldova: ameninţări, tendinţe şi recomandări de reabilitare, Monografie, Academia de Ştiinţe a Moldovei, Institutul de Zoologie al Academiei de Ştiinţe a Moldovei, Chişinău, ISBN 978-9975-89-070-0, 1-343. (in Romanian)

13. Davideanu G., Moșu A., Davideanu A. and Miron A., 2008 - Ihtiofauna râului Prut, Societatea Ecologică pentru Protecţia şi Studierea Florei şi Faunei Sălbatice AQUATERRA, Societatea Bioremedierii Ecosistemelor Acvatice şi Umede „Euribiont”, 80. (in Romanian)

14. Engelman L., 2005 - Discriminant analysis, Systat 11. Statistics manual, I, 301-359.

15. Foster K., Bower L. and Piller K., 2015 - Getting in shape: habitat-based morphological divergence for two sympatric fishes, Biological Journal of the Linnean Society, 114, 152-162, https://doi.org/10.1111/bij.12413.

16. Geiger M. F. and Schliewen U. K., 2010 - Gymnocephalus ambriaelacus, a new species o ruffe from Lake Ammersee, southern Germany, Spixiana, 33, 119-137.

17. Györe K., Józsa V., Lengyel P. and Gál D., 2013 - Fish faunal studies in the Körös River system, AACL Bioflux, 6, 1, 34-41.

18. Heyman H. and Noble A. C., 1989 - Comparison of Canonical Variate and Principal Components analyses of Wine Descriptive Analysis Data, Journal of Food Science, 54, 5, 1355 1358.

19. Holčík J. and Hensel K., 1974 - A new species of Gymnocephalus (Pisces: Percidae) from the Danube, with Remarks on the Genus, Copeia, 2, 471-486. 
20. Karakousis Y., Triantaphyllidis C. and Economidis P. S., 1991 - Morphological variability among seven populations of brown trout, Salmo trutta L., in Greece, Journal of Fish Biology, 38, 807-817.

21. Klingenberg C. P., 2011 - MorphoJ: an integrated software package for geometric morphometrics, Molecular ecology resources, 11, 353-357.

22. Kottelat M. and Freyhof J., 2007 - Handbook of European freshwater dishes, Publications Kottelat, Cornol, 646.

23. Kováč V., 1994 - Early ontogeny of three Gymnocephalus species (Pisces: Percidae): reflections on the evolution of the genus, Environmental Biology of Fishes, 40, 241-253.

24. Năstase A. and Oțel V., 2016 - Researches on the fish fauna in some SCIs Natura 2000 from Romania, AACL Bioflux, 9, 3, 527-540.

25. Năstase A., Oțel V. and Năvodaru I., 2017 - Ecological status of fish fauna in arms of the Danube Delta (Danube Delta Biosphere Reserve, Romania) at the beginning of the third millennium, Acta Zoologica Bulgarica, 69, 3, 349-360.

26. Nuță A. M., Diaconescu Ș., Dănălache T., Filep R. and Nicolae C. G., 2016 - Research of dynamic ichtyofauna from Comana Nature Park, Agro Life Scientific Journal, 5, 2, 108-111.

27. Oțel V., 2007 - Atlasul peştilor din Rezervaţia Biosferei Delta Dunării, Edit. Centrul de Informare Tehnologică Delta Dunării al I. N. C. D. D. D., Tulcea, 481. (in Romanian)

28. Oțel V. and Năstase A., 2010 - Researches on ichthyofauna in the Natura 2000 sites from Banat (Romania), Analele Ştiinţifice ale Institutului - Delta Dunării, 16, 33-38.

29. Oţel V., Bănărescu P., Nalbant T. and Baboianu C., 1993 - Rezultatele investigaţiilor ihtiologice din teritoriul Rezervaţiei Biosferei Delta Dunării în anul 1992, Analele Ştiinţifice ale Institutului - Delta Dunării, 145-162. (in Romanian)

30. Povž M., Mracovčić M. and Kerovec M., 1997 - The first find of balon’s ruffe (Gymnocephalus baloni) in the river Drava in Slovenia and Croatia, Folia Zoologica, 46, 2, 189-190.

31. Ratschan C., 2012 - Verbreitung, Habitatwahl und Erhaltungszustand des Donaukaulbarsches (Gymnocephalus baloni Holčík and Hensel, 1974) in Österreich, Österreichs Fischerei, 65, 218231. (in German)

32. Rohlf F. J., 2016a - TpsDig2 version 2.26 (Tps_Digitize), http://life.bio.sunysb.edu/morph/).

33. Rohlf F. J., 2016b - TpsRelw version 1.67 (Tps_Relative Warps). http://life.bio.sunysb.edu/morph

34. Rohlf F. J., 2016c - TpsUtil version 1.70 (Tps_Utility program). http://life.bio.sunysb.edu/morph

35. Samaradivakara S. P., Hirimuthugoda N. Y., Gunawardana R. H. A. N. M., Illeperuma R. J., Fernandopulle N. D., De Silva A. D. and Alexander P. A. B. D., 2012 - Morphological variation of four Tilapia populations in selected reservoirs in Sri Lanka, Tropical Agricultural Research, 23, 2, 105-116.

36. Shao Y., Wang J., Qiao Y., He Y. and Cao W., 2007 - Morphological Variability Between Wild Populations and Inbred Stocks of a Chinese Minnow, Gobiocypris rarus, Zoological Science, 24, 1094-1102, https://doi.org/10.2108/zsj.24.1094

37. Specziár A. and Vida A., 1995 - Comparative study of Gymnocephalus cernuus (Linnaeus, 1758) and G. baloni Holčík and Hensel, 1974 (Pisces, Percidae), Miscellanea Zoologica Hungarica, 10, 103-116.

38. Stepien C. A. and Haponski A. E., 2015 - Taxonomy, distribution, and evolution of percid fishes, Chapter 1, 3-60, in Biology and Culture of Percid Fishes-Principles and Practices, Kestlemont P., Dabrowski K. and Summerfelt R. C. (eds), Springer, ISBN 978-94-917-7227-3, http://www.utoledo.edu/nsm/lec/research/glgl/publications/StepienHaponski2015_PercidCh1.

39. Telcean I. C. and Cupşa D., 2009 - Fish fauna from the lowland Mureş River (Romania) and the floodplain natural park area (western Romania), Analele Universității din Oradea, Fascicula Biologie, XVI, 1, 131-135.

40. Telcean I. C. and Cupșa D., 2012 - Threatened and rare fishes from upper Tisa Valley and its Romanian left shore tributaries (north-western Romania), Pisces Hungarici, 6, 87-94. 
41. Tsyba A. O. and Kokodiy S. V., 2017 - The morphological characteristics of the Danube ruffe, Gymnocephalus baloni (Perciformes, Percidae) in the upper reaches of the Dnipro River, Ukraine, Vestnik Zoologii, 51, 1, 79-82.

42. Turan C., 1999 - A note on the examination of morphometric differentiation among fish populations: the truss system, Turkish Journal of Zoology, 23, 259-263.

43. Wimberger P. H., 1992 - Plasticity of fish body shape. The effects of diet, development, family and age in two species of Geophagus (Pisces, Cichlidae), Biological Journal of the Linnean Society, 45, 3, 197-218, https://doi.org/10.1111/j.1095-8312.1992.tb00640.x 